\title{
Resection of the Dorsal Sector of the Liver: The Anterior Transhepatic Approach
}

\author{
Heithem Jeddou, MD, Stylianos Tzedakis, MD, Laurent Sulpice, MD, PhD, and Karim Boudjema, MD, PhD \\ Department of Hepatobiliary and Digestive Surgery, Pontchaillou University Hospital, Rennes, France
}

\begin{abstract}
Background. As defined by Couinaud et al. and Takasaki et al., the dorsal sector (DS) or caudate area is the portion of liver parenchyma located between the retrohepatic inferior vena cava (IVC) and the main hepatic veins confluence. ${ }^{1,2}$ Its complete resection, usually approached by right- or left-sided liver parenchymotomy, remains technically challenging. ${ }^{3,4}$ Moreover, these approaches usually fail to properly expose the middle (paracaval portion) and most cranial portion (i.e. behind the hepatic vein confluence) of the DS. ${ }^{5}$

Methods. We report the case of a 50-year-old woman presenting with a unique metachronous hepatic metastasis of a colorectal adenocarcinoma. The 40-mm lesion was located behind the confluence of the three main hepatic veins, in front of the IVC, and extended caudally behind the hepatic hilum, thereby occupying the paracaval portion of the DS. The tumor board decision was surgical resection.

Results. The procedure included complete anatomic resection of the DS using an anterior transhepatic approach by opening the liver midplane along the Cantlie line. A well-tolerated continuous pedicle and IVC clamping of $30 \mathrm{~min}$ was used. The duration of the surgery was $120 \mathrm{~min}$, and the blood loss was $200 \mathrm{~mL}$. The patient was discharged on postoperative day 5. R0 resection was achieved and the patient was free of disease at 1 year post-surgery.
\end{abstract}

Electronic supplementary material The online version of this article (https://doi.org/10.1245/s10434-019-07376-x) contains supplementary material, which is available to authorized users.

(C) Society of Surgical Oncology 2019

First Received: 8 February 2019;

Published Online: 10 June 2019

K. Boudjema, MD, PhD

e-mail: karim.boudjema@chu-rennes.fr
Conclusion. Anatomic resection of the DS using a transparenchymal anterior approach is a safe and feasible procedure especially suitable for voluminous tumors. It allows the control of all major vascular structures narrowing the lesion, and facilitates an $\mathrm{R} 0$ resection.

\section{REFERENCES}

1. Couinaud C. Surgical anatomy of the liver. Several new aspects. Chirurgie. 1986;112(5):337-42. [in French].

2. Takasaki K. Glissonean pedicle transection method for hepatic resection: a new concept of liver segmentation. $J$ Hepatobiliary Pancreat Surg. 1998;5(3):286-91.

3. Chaib E, Ribeiro MA Jr, Silva Fde S, et al. Caudate lobectomy: tumor location, topographic classification, and technique using right- and left-sided approaches to the liver. Am J Surg. 2008;196(2):245-51.

4. Wang Y, Zhang LY, Yuan L, et al. Isolated caudate lobe resection for hepatic tumor: surgical approaches and perioperative outcomes. Am J Surg. 2010;200(3):346-51.

5. Kumon M. Anatomical study of the caudate lobe with special reference to portal venous and biliary branches using corrosion liver casts and clinical application. Liver Cancer. 2017;6(2):161-70.

Publisher's Note Springer Nature remains neutral with regard to jurisdictional claims in published maps and institutional affiliations. 